\title{
Efectos de un programa de ejercicio físico de 12 semanas en sujetos con revascularización coronaria o postangioplastia transluminal percutánea
}

\author{
Mirary Mantilla-Morrón ${ }^{\mathrm{a}, *}$, Miguel Urina-Triana ${ }^{\mathrm{b}}$, Yaneth Herazo-Beltrán ${ }^{\mathrm{c}}$ \\ y Daniela Urina-Jassir ${ }^{d}$
}

\author{
a Programa de Fisioterapia, Universidad Simón Bolívar, Barranquilla, Colombia \\ ${ }^{b}$ Centro de Investigaciones Cardiodiagnóstico SA/Fundación del Caribe para la Investigación Biomédica, \\ Universidad Simón Bolívar, Barranquilla, Colombia \\ c Facultad de Medicina, Universidad Simón Bolívar, Barranquilla, Colombia \\ d Centro de Investigaciones Cardiodiagnóstico SA/Fundación del Caribe para la Investigación Biomédica, Barranquilla, Colombia
}

Recibido el 11 de diciembre de 2015; aceptado el 25 de julio de 2016

Disponible en Internet el 4 de octubre de 2016

\section{PALABRAS CLAVE \\ Enfermedad \\ Coronaria; \\ Rehabilitación; \\ Ejercicio}

\begin{abstract}
Resumen
Objetivo: Determinar los efectos de un programa de ejercicio físico sobre las características antropométricas y la capacidad funcional postcirugía de revascularización coronaria o postangioplastia coronaria transluminal percutánea.

Métodos: Estudio clínico no controlado, de antes y después, en 49 pacientes postcirugía de revascularización coronaria o angioplastia coronaria percutánea. El índice de masa corporal, perímetro abdominal, índice de cintura cadera, perímetro de la cadera y la capacidad cardiorrespiratoria a través de la caminata de los seis minutos fueron evaluados antes y al finalizar el programa de ejercicio físico. Este estuvo conformado por ejercicios aeróbicos continuos, de resistencia muscular, equilibrio y coordinación, durante 3 días a la semana, durante 12 semanas. Resultados: Con relación al perímetro abdominal en las mujeres cambió de $91,4 \mathrm{~cm}$ a $88,4 \mathrm{~cm}$ $(p=0,003)$ y en los hombres de $93,6 \mathrm{~cm}$ a 89,8 $(p=0,000)$. Se logró un incremento significativo de la distancia caminada de 330 a 436 metros $(p=0,000)$; del consumo energético de 3,4 a 4,1 MET $(\mathrm{p}=0,000)$; y la máxima capacidad de absorber, transportar y consumir el oxígeno, de 12 a $14 \mathrm{ml} / \mathrm{Kg} / \mathrm{min}$.

Conclusiones: El programa de ejercicio físico que fue utilizado en este estudio, pudo en todos los sujetos incrementar la capacidad cardiorrespiratoria y la tolerancia al ejercicio, y disminuir el perímetro abdominal. Los efectos benéficos obtenidos cambian el pronóstico de la enfermedad coronaria y disminuyen los factores de riesgo metabólicos.

(c) 2016 Sociedad Colombiana de Cardiología y Cirugía Cardiovascular. Publicado por Elsevier España, S.L.U. Este es un artículo Open Access bajo la licencia CC BY-NC-ND (http:// creativecommons.org/licenses/by-nc-nd/4.0/).
\end{abstract}

* Autor para correspondencia.

Correo electrónico: mmantilla2@unisimonbolivar.edu.co (M. Mantilla-Morrón).

http://dx.doi.org/10.1016/j.rccar.2016.07.008

0120-5633/@ 2016 Sociedad Colombiana de Cardiología y Cirugía Cardiovascular. Publicado por Elsevier España, S.L.U. Este es un artículo Open Access bajo la licencia CC BY-NC-ND (http://creativecommons.org/licenses/by-nc-nd/4.0/). 


\section{KEYWORDS}

Coronary disease; Rehabilitation;

Exercise

\section{Effects of a 12-week exercise program for individuals with coronary revascularization or after percutaneous transluminal coronary angioplasty}

\begin{abstract}
Motivation: To determine the effects of a physical exercise program in the anthropometric characteristics and postsurgical functional capacity after coronary revascularization or percutaneous transluminal coronary angioplasty.

Methods: Uncontrolled clinical before and after study, in 49 patients who has undergone coronary revascularization or percutaneous transluminal coronary angioplasty. Body mass index, abdominal girth, waist-to-hip ratio, hip perimeter and cardiorespiratory function were assessed by means of the six-minute walk test before and after the physical exercise program. It consisted of continuous aerobic, muscle resistance, balance and coordination exercises three times a week during a total of 12 weeks.

Results: Abdominal girth changed from $91.4 \mathrm{~cm}$ to $88.4 \mathrm{~cm}(\mathrm{p}=0.003)$ in women and from $93.6 \mathrm{~cm}$ to $89.8 \mathrm{~cm}(p=0.000)$. A significant increase of the walked distance was achieved, from 330 to 436 meters $(p=0.000)$; energy use from 3.4 to 4.1 MET $(p=0.000)$; and maximum capacity of absorbing, transporting and consuming oxygen, from 12 to $14 \mathrm{ml} / \mathrm{Kg} / \mathrm{min}$.

Conclusion: The physical activity program used in this study contributed to an increase in all participants of the cardiorespiratory function and exercise tolerance, and to a decrease in abdominal girth. These beneficial effects change the prognosis of the coronary disease and reduce the metabolic risk factors.

(c) 2016 Sociedad Colombiana de Cardiología y Cirugía Cardiovascular. Published by Elsevier España, S.L.U. This is an open access article under the CC BY-NC-ND license (http:// creativecommons.org/licenses/by-nc-nd/4.0/).
\end{abstract}

\section{Introducción}

Las enfermedades cardiovasculares ocupan el primer lugar de la morbimortalidad en el mundo ${ }^{1}$, y para el año 2020 los más afectados serán los países de medianos y bajos ingresos $^{2}$. La revascularización cardiaca a través de la angioplastia o por el injerto de hemoductos en las arterias coronarias, ha demostrado ser eficaz reduciendo la severidad y las complicaciones del síndrome coronario, modificando considerablemente las estancias hospitalarias y el tiempo de reincorporación del sujeto a sus actividades de la vida cotidiana; estas dos intervenciones, además, pueden reducir los síntomas tales como: la angina y la disnea, mejorando así la capacidad para llevar a cabo el entrenamiento físico $^{3,4}$.

La evidencia científica ha demostrado que el ejercicio físico controlado o cualquier otra forma de actividad física, se asocia con una menor morbilidad y con una reducción en todas las causas de la mortalidad de un 20 a $30 \%^{5-7}$, por ello, es un imperativo la remisión a los programas controlados del ejercicio físico después de la revascularización coronaria.

En otros estudios se ha demostrado que existe una fuerte relación entre una mayor capacidad de ejercicio y un pronóstico favorable en los pacientes con la enfermedad arterial coronaria $^{8,9}$. Los programas de rehabilitación cardiaca, son muy importantes para la prevención secundaria y una clave para lograrla es el ejercicio programado, el cual mejora la tolerancia física durante las actividades cotidianas y aumenta el consumo máximo de oxígeno $\left(\mathrm{VO}_{2}\right.$ máx.), que, además, de ser un predictor de supervivencia en los pacientes cardiacos, también se utiliza para prescribir el ejercicio de manera segura, individualizada y efectiva, lo que conlleva a plantear objetivos reales y alcanzables ${ }^{10}$.
De acuerdo con las investigaciones anteriores, el objetivo de la rehabilitación cardiaca es complementar el tratamiento cardiológico, a través de un conjunto de actividades relacionadas con la educación y la promoción de un estilo de vida activo para reducir el riesgo cardiovascular y mejorar el pronóstico y la calidad de vida ${ }^{11,12}$.

Las estrategias usadas por la rehabilitación cardiaca mejoran la capacidad funcional y el nivel de condición física de los pacientes después de una cirugía cardiaca al modificar las alteraciones del sistema músculo-esquelético presentes como: la atrofia muscular, los cambios en el tipo de fibra muscular, el desajuste en la demanda-perfusión y la disfunción metabólica ${ }^{13,14}$.

Por otra parte, el ejercicio físico aumenta el consumo máximo del oxígeno, incrementa la capacidad física, produce adaptaciones en la estructura y función del músculo-esquelético, mejora el flujo sanguíneo periférico y la función endotelial, incrementa el tono vagal, disminuye el tono simpático, baja el riesgo de arritmias y disminuye las citosinas proinflamatorias ${ }^{15,16}$, produciendo una mejora en la composición corporal a largo plazo, al lograr reducir la masa grasa y aumentar la masa magra, además, el ejercicio es capaz de modificar los factores de riesgo metabólicos, y ha demostrado disminuir la resistencia a la insulina en particular en los sujetos con síndrome metabólico en el que el perímetro abdominal representa el mayor indicador de distribución de grasa abdominal y si se logra reducirlo se impactará en su pronóstico ${ }^{17}$.

Con base en lo anteriormente expuesto el propósito del presente estudio fue determinar los efectos del programa de ejercicio físico continúo sobre las características antropométricas y la capacidad funcional en los sujetos después 
de ser sometidos a la revascularización coronaria quirúrgica o por la angioplastia percutánea.

\section{Material y métodos}

Se realizó un estudio clínico no controlado, de antes y después, para evaluar el impacto que sobre las características antropométricas y la capacidad funcional tiene la intervención con un programa de ejercicios físicos de 12 semanas sobre los pacientes postcirugía de la revascularización coronaria o la angioplastia coronaria transluminal percutánea, que ingresaron al departamento de rehabilitación cardiaca de la IPS Cardiodiagnóstico SA en la ciudad de Barranquilla en el período comprendido entre enero a diciembre de 2014.

\section{Selección y descripción de los participantes}

Durante el período de observación, ingresaron al servicio de rehabilitación cardiaca un total de 60 individuos. Se seleccionaron a los sujetos que cumplieron los siguientes criterios para su inclusión: 1. Haber recibido la revascularización coronaria por cirugía con colocación de hemoductos o con la angioplastia percutánea (el procedimiento debería tener al menos 30 días de haber sido efectuado). 2. Capacidad funcional igual o mayor a 4 METS según criterios de la New York Heart Association ${ }^{18}$, obtenida mediante una prueba ergométrica, y 3. Firma en forma voluntaria del consentimiento para participar en el estudio.

Como criterios de la exclusión se tuvieron en cuenta los siguientes: 1. Limitaciones osteomusculares para realizar ejercicio físico. 2. Arritmias ventriculares no tratadas. 3. Presencia de derrame pericárdico residual. 4. Historia clínica de claudicación intermitente, y 5 . Fracción de eyección < $30 \%$ medida por ecocardiograma.

Fueron escogidos para participar 49 (n) sujetos de la población total $(\mathrm{N}=60)$ (dos sujetos no cumplieron los criterios de inclusión y nueve no lo hicieron por razones de distancia $(n=3)$ o económicas $(n=6)$. De los sujetos seleccionados, 28 habían recibido angioplastia percutánea y 21 revascularización coronaria por cirugía con colocación de hemoductos. A todos los sujetos se le evaluó su historia clínica previa con relación a la hipertensión arterial sistémica, la diabetes mellitus tipo 2 y se revisaron los tratamientos que estaban recibiendo a la fecha.

La evaluación y prescripción del ejercicio fue supervisada y asistida por un grupo multidisciplinario con la participación de un fisioterapeuta rehabilitador cardiaco y un cardiólogo rehabilitador.

\section{Medidas de resultados}

Al inicio y al final del programa del ejercicio físico se evaluaron las características sociodemográficas, antropométricas, fisiológicas, información clínica, además, datos sobre los factores de riesgo y estado funcional; datos como: triglicéridos, colesterol HDL y glicemia en ayunas se tomaron de la historia clínica del sujeto.

La capacidad física se evaluó mediante la Prueba de Caminata de 6 Minutos, una prueba de ejercicio submáximo de campo, indicador de la capacidad funcional y predictor del consumo máximo de oxígeno $\left(\mathrm{VO}_{2}\right.$ máx. $)$ a partir de la distancia recorrida ${ }^{3}$, los pacientes fueron instruidos para caminar en la medida de lo posible por un pasillo plano de 30 metros, la prueba fue limitada por los síntomas, si era necesario la prueba se interrumpiría si había angina de pecho, disnea severa, mareo, dolor músculo-esquelético; se le permitió descansar a los pacientes si era necesario y que reiniciaran cuando les fuese posible, se tuvo en cuenta los períodos de descanso, su duración y síntomas. Durante la prueba los pacientes recibieron estímulo estandarizado cada minuto, la distancia total recorrida se midió al metro más cercano; antes de iniciar, durante la caminata y al final de la prueba se tomaron datos como: la frecuencia cardiaca, la presión arterial, la saturación de oxígeno, y la percepción subjetiva del esfuerzo según la escala de Borg en posición sedente; al finalizar la prueba se registró la distancia total caminada. Los resultados de la Prueba Caminata de 6 Minutos se registraron como valor absoluto en metros, teniendo en cuenta las variables antropométricas (edad, sexo, peso y altura) de acuerdo con la ecuación de referencia propuesta por Troosters, y para la predicción del $\mathrm{VO}_{2}$, se usó la ecuación propuesta por el Colegio Americano de Medicina del Deporte: $\mathrm{VO}_{2}$ máx. $=0,15 \mathrm{ml} / \mathrm{kg} /$ $\mathrm{min}^{*}$ (Distancia/tiempo) + 3,5 $\mathrm{ml} / \mathrm{kg} / \mathrm{min}^{19}$.

El peso corporal se determinó mediante una balanza digital de bioimpedancia de los pies y las manos con capacidad de $200 \mathrm{~kg}$ y precisión de $100 \mathrm{~g}\left(\mathrm{OMRON}^{\circledR}\right.$, Modelo HBF-500 INT); durante la medición el paciente estuvo descalzo con un mínimo de ropa, los brazos a lo largo del cuerpo y mirando hacia el frente. La talla fue medida a través de un tallímetro sin zapatos y en inspiración profunda. El índice de masa corporal, se calculó dividiendo el peso corporal por la talla elevada al cuadrado $\left(\mathrm{kg} / \mathrm{m}^{2}\right)$ y teniendo como criterios los de la Organización Mundial de la Salud, la obesidad fue definida con el índice de masa corporal IMC $\geq 30 \mathrm{~kg} / \mathrm{m}^{2}$ y sobrepeso $(I M C) \geq 25$ a $29,9 \mathrm{~kg} / \mathrm{m}^{2},{ }^{20}$.

El perímetro abdominal se tomó justo por encima de la cresta ilíaca anterosuperior con el paciente en posición de pie, con una cinta métrica flexible, extensible milimetrada con ancho máximo de $5 \mathrm{~mm}$ y precisión de $0,1 \mathrm{~cm}$; se consideró un perímetro abdominal de riesgo $\geq 90 \mathrm{~cm}$ en hombres y en mujeres $\geq 80 \mathrm{~cm}^{21}$; el índice de cintura cadera se estimó a partir de la división del perímetro abdominal y el perímetro de la cadera, medido en la zona del trocánter mayor del fémur ${ }^{22}$.

La percepción subjetiva de esfuerzo del sujeto y el ajuste de la intensidad del ejercicio se definió con la Escala de Borg ${ }^{23}$. Se monitorizó la frecuencia cardiaca con un pulsioxímetro Pulox Po200. La presión arterial sistólica y la presión arterial diastólica fueron evaluadas con un esfigmomanómetro aneroide, previamente calibrado. Esta se registró antes, durante y 10 minutos después de finalizar el ejercicio físico. Se consideró que para iniciar el ejercicio físico el paciente debía tener valores de la presión arterial sistólica < $135 \mathrm{~mm}$ $\mathrm{Hg}$ y la presión arterial diastólica de $<85 \mathrm{~mm} \mathrm{Hg}$; si durante el desarrollo de las sesiones el paciente alcanzaba presiones arteriales de $180 \mathrm{~mm} \mathrm{Hg} / 110 \mathrm{~mm} \mathrm{Hg}$, se interrumpía la sesión de trabajo ${ }^{24}$.

\section{Programa de intervención}

El programa del ejercicio estuvo conformado por: los ejercicios aeróbicos, de resistencia muscular, equilibrio y 
Tabla 1 Características sociodemográficas de los pacientes

\begin{tabular}{lll}
\hline Variables & Frecuencia & Porcentaje \\
\hline Sexo & 19 & \\
Femenino & 30 & 38,8 \\
Masculino & & 61,2 \\
Estado Civil & 27 & \\
Con pareja & 22 & 55,1 \\
Sin pareja & & 44,9 \\
Estrato socioeconómico & & \\
Estrato 1 & 22 & 44,9 \\
Estrato 2 & 7 & 14,3 \\
Estrato 3 & 9 & 18,4 \\
Estrato 4 & 7 & 14,3 \\
Estrato 5 & 1 & 2,0 \\
Estrato 6 & 3 & 6,1 \\
Aseguramiento & & \\
Contributivo & 28 & 57,1 \\
Subsidiado & 15 & 30,6 \\
Vinculado & 6 & 12,2 \\
\hline
\end{tabular}

coordinación en forma continúa, durante 3 días a la semana, durante

12 semanas. El ejercicio aeróbico consistió en 3 sesiones en días alternos por semana, con una duración de 30 a 60 minutos por sesión, con una intensidad entre el 40 al $80 \%$ del consumo máximo de oxígeno de reserva, monitorizada con la escala de esfuerzo percibido Borg; la progresión del ejercicio fue individualizada y se realizaron incrementos de 5 minutos por día hasta alcanzar los 60 minutos y en cuanto al aumento de la resistencia en las máquinas fue de 1 nivel por semana, hasta alcanzar los 10 niveles. En el programa de resistencia muscular se utilizaron pesas libres en una repetición con incremento o disminución de la carga de 2,5 a 5 libras, hasta determinar el peso máximo a levantar. Una repetición máxima se consideró como válida con una extensión completa del grupo muscular utilizado, sin sustituciones musculares ${ }^{25}$.

El proyecto fue aprobado por el Comité Ético de la Universidad Simón Bolívar. Los datos se analizaron con el software SPSS versión 17 (licencia de la Universidad Simón Bolívar). Mediante la prueba Kolmogórov-Smirnov (K-S) se verificó la normalidad de los datos pre y post del grupo de estudio. La diferencia de medias del pre y el post se determinó a través de las pruebas $t$ de Student y de rangos con signos de Wilcoxon, considerándose un valor de $\mathrm{p} \leq 0,05$ como significativo.

\section{Resultados}

Las características de los 49 sujetos de estudio se muestran en la tabla 1. En este estudio el $61,2 \%$ fueron hombres y el $38,8 \%$ mujeres, con promedio de edad para la población estudiada de $63 \pm 8,9$ años.

El $55 \%$ vivía en pareja el $44,9 \%$ pertenecía a un estrato bajo y el $57,1 \%$ estaba afiliado al régimen de salud contributivo. 28 pacientes habían recibido angioplastia percutánea y 21 revascularización coronaria por cirugía con colocación de hemoductos.
Tabla 2 Características de salud de los pacientes sujetos de estudio

\begin{tabular}{lll}
\hline Variables & Frecuencia & Porcentaje \\
\hline Patologías asociadas & & \\
Hipertensión arterial sistémica & 41 & 83,7 \\
Diabetes mellitus tipo 2 & 33 & 66 \\
\hline
\end{tabular}

Tabla 3 Promedios de las características metabólicas al inicio del estudio

\begin{tabular}{|c|c|c|}
\hline Variables & Media (DE) & Rango \\
\hline Triglicéridos (mg/dl) & $145,3(44,3)$ & $86-264$ \\
\hline $\begin{array}{l}\text { Colesterol HDL en mujeres } \\
(\mathrm{mg} / \mathrm{dl})\end{array}$ & $50,6(18,9)$ & $21-93$ \\
\hline $\begin{array}{l}\text { Colesterol HDL en hombres } \\
(\mathrm{mg} / \mathrm{dl})\end{array}$ & $41,1(11,2)$ & $30-73$ \\
\hline Glicemia (mg/dl) & $120,8(43,1)$ & $59-285$ \\
\hline Presión sistólica (mm Hg) & $123,5(18,8)$ & $76-171$ \\
\hline Presión diastólica (mm Hg) & $66,8(13,1)$ & $42-112$ \\
\hline
\end{tabular}

El 40,8\% recibía bloqueadores de canales de calcio, (n: $20)$; el 83,67\% betabloqueadores ( $n: 41)$; el 93,87 estatinas, $(n=46)$; el 67,34 hipoglucemiantes orales $(n=33)$; el $8,1 \%$ insulina $(n=4)$; y el 32,65 bloqueadores de los receptores de angiotensina ॥ $(n=16)$.

En cuanto a las características de la salud, el 83,7\% tenían hipertensión arterial sistémica y el $66 \%$ diabetes mellitus tipo 2 (tabla 2).

En la tabla 3 se observan los promedios de las características metabólicas al inicio del estudio, resaltando que los promedios del colesterol HDL están en los límites de riesgo tanto en los hombres como en las mujeres.

Se observan diferencias significativas $(p=<0,05)$ al comparar los valores antes y después de la intervención de las características antropométricas de los participantes. El mayor cambio se obtuvo en el perímetro abdominal tanto en hombres como en las mujeres; en esta última población, al inicio del programa un promedio de $91,4 \mathrm{~cm}$, logrando disminuir a $88,4 \mathrm{~cm}$ al final de la intervención (tabla 4).

En la tabla 5 se muestra el incremento significativo de la distancia caminada durante la Prueba de Caminata de 6 Minutos, de 330 a 436 metros $(p=0,000)$. Así mismo, el consumo energético de 3,4 a 4,1 MET $(p=0,000)$; y la máxima capacidad de absorber, transportar y consumir el oxígeno, de 12 a $14 \mathrm{ml} / \mathrm{kg} / \mathrm{min}$.

\section{Discusión}

Los resultados del presente estudio confirman lo reportado por Aikawa ${ }^{26}$, en cuanto a los beneficios del ejercicio físico en la salud y el bienestar de las personas a quienes le han realizado la cirugía de revascularización miocárdica o la angioplastia coronaria percutánea, contribuyendo al retorno de una vida activa y a la prevención de problemas cardiacos futuros e incluso la muerte; esto convierte a la rehabilitación cardiaca en parte esencial de la atención de salud de dichos pacientes, siendo su elemento central el programa de ejercicio físico ${ }^{27}$. 
Tabla 4 Medias de las características antropométricas al inicio y al final de la intervención en la población general

\begin{tabular}{llll}
\hline & & Población general & \\
\cline { 2 - 4 } & Pre-intervención & Post-intervención & Valor de $\mathrm{p}$ \\
\hline Perímetro de cintura $(\mathrm{cm})$ & $92,7 \pm 10,7$ & $89,2 \pm 10,6$ & 0,000 \\
Perímetro de cadera $(\mathrm{cm})$ & $96,3 \pm 8,6$ & $94,6 \pm 8,1$ & 0,002 \\
Índice cintura cadera $(\mathrm{m})$ & $0,96 \pm 0.07$ & $0,94 \pm 0,07$ & 0,001 \\
Índice de Masa Corporal $\left(\mathrm{kg} / \mathrm{m}^{2}\right)$ & $24,2 \pm 3,7$ & $23,7 \pm 3,3$ & 0,03 \\
\hline
\end{tabular}

Tabla 5 Medias de las características antropométricas al inicio y al final de la intervención en hombres y mujeres

\begin{tabular}{|c|c|c|c|c|c|c|}
\hline & \multicolumn{3}{|c|}{ Hombres } & \multicolumn{3}{|c|}{ Mujeres } \\
\hline & $\begin{array}{l}\text { Pre- } \\
\text { intervención }\end{array}$ & $\begin{array}{l}\text { Post- } \\
\text { intervención }\end{array}$ & $\begin{array}{l}\text { Valor } \\
\text { de } p\end{array}$ & $\begin{array}{l}\text { Pre- } \\
\text { intervención }\end{array}$ & $\begin{array}{l}\text { Post- } \\
\text { intervención }\end{array}$ & $\begin{array}{l}\text { Valor } \\
\text { de } p\end{array}$ \\
\hline Perímetro de cintura $(\mathrm{cm})$ & $93,6 \pm 10,6$ & $89,8 \pm 9,8$ & 0,000 & $91,4 \pm 11,1$ & $88,4 \pm 11,9$ & 0,003 \\
\hline Perímetro de cadera $(\mathrm{cm})$ & $96,7 \pm 8,7$ & $94,8 \pm 8,3$ & 0,001 & $95,7 \pm 8,6$ & $94,2 \pm 8$ & 0,002 \\
\hline Índice cintura cadera $(\mathrm{m})$ & $0,97 \pm 0,06$ & $0,94 \pm 0,08$ & 0,007 & $0,95 \pm 0,06$ & $0,94 \pm 0,06$ & 0,06 \\
\hline Índice de Masa Corporal $\left(\mathrm{kg} / \mathrm{m}^{2}\right)$ & $24,5 \pm 3,8$ & $24,1 \pm 3,3$ & 0,2 & $23,7 \pm 3,7$ & $23,1 \pm 3,4$ & 0,02 \\
\hline
\end{tabular}

Tabla 6 Valores promedios de la capacidad funcional al inicio y al final de la intervención

\begin{tabular}{lcccc}
\hline Variables & Medias Pre-intervención & Medias post-intervención & Diferencias de medias & Valor de $\mathrm{p}$ \\
\hline Disnea (Borg) & $1,7(1,3)$ & $1,4(1)$ & $0,3(1,2)$ & $0,4(1,4)$ \\
Esfuerzo percibido & $2,3(1,4)$ & $1,9(1)$ & & 0,12 \\
(fatiga) & & & $-105,4(89)$ & 0,07 \\
Metros recorridos & $330(114)$ & $436(79)$ & $-0,18(0,6)$ & 0,000 \\
Minutos caminando & $5,8(0,6)$ & $6(0)$ & $-2,4(2,2)$ & 0,03 \\
VOmáx & $12(2,6)$ & $14(0,7)$ & $-0,68(0,6)$ & 0,000 \\
(ml/kg/min) & $3,4(0,7)$ & $4,1(0,5)$ & $-46,4(45)$ & 0,000 \\
MET & $223,6(68)$ & $270,1(75)$ & $-0,72(0,8)$ & 0,000 \\
Kilo/Calorías & $3,73(1,1)$ & $4,45(1,2)$ & & 0,000 \\
Kilo/Calorías & & & & \\
Esfuerzo & & &
\end{tabular}

Se ha apreciado que el parámetro antropométrico con mayor sensibilidad al programa de los ejercicios prescritos fue el perímetro abdominal, lo cual coincide con los hallazgos de otros autores ${ }^{28}$, ratificando que el ejercicio regular es uno de los componentes centrales en la pérdida de peso. La disminución de grasa abdominal en los pacientes participantes del estudio incide en la disminución de la resistencia a la insulina, resultado positivo si se tiene en cuenta que un porcentaje alto de ellos presentan diabetes tipo 2 .

En cuanto a los metros recorridos durante la Prueba de 6 Minutos se observó un incremento significativo después de la cirugía de revascularización; este resultado es similar al reportado por Aikawa ${ }^{26}$; en su estudio los sujetos del estudio recorrieron un promedio de $\mathbf{4 7 9}$ metros antes de la intervención y alcanzaron después del programa de la rehabilitación cardiaca, 545 metros; los autores reconocen la distancia recorrida durante la Prueba de 6 Minutos como un indicador adecuado para evaluar la capacidad de los pacientes en la realización de sus actividades diarias.

Se evidenció en este estudio que un programa de ejercicios incrementa la máxima capacidad de absorber, transportar y consumir el oxígeno, aunque no alcanza los valores de la población normal, resultados también demostrados en el estudio de Jeline ${ }^{3}$, de 12,6 $\pm 1,0 \mathrm{ml} / \mathrm{kg} / \mathrm{min}$, se logró un cambio significativo a $13,3 \pm 1.3 \mathrm{ml} / \mathrm{kg} / \mathrm{min}$; estos resultados indican una mejoría del pronóstico de los pacientes, toda vez que un incremento de $1 \mathrm{ml} / \mathrm{kg} / \mathrm{min}$ en el consumo de oxígeno resulta en un $10 \%$ de mejoría como lo expresa Najafi ${ }^{28}$. El consumo del oxígeno ha sido considerado como el predictor más importante de la mortalidad futura ${ }^{19}$, en este sentido, lo obtenido en esta investigación indica que en los pacientes se logró un aumento de su función cardiovascular y en las adaptaciones musculares inducidas por el programa del ejercicio físico (tabla 6)

El programa de intervención aplicado a los participantes del presente estudio fue realizado en un centro de rehabilitación cardiaca, cuya característica principal es un plan de ejercicio físico estructurado, planificado y supervisado, en comparación con un programa ejecutado en el hogar basado en la actividad física libre como $\operatorname{caminar}^{27}$, aunque la revisión sistemática de Taylor $^{29}$ confirma que ambos programas son igualmente eficaces para mejorar los parámetros 
clínicos en los pacientes después de una revascularización, la elección entre los dos programa depende de la preferencia del paciente.

\section{Conclusiones}

El programa del ejercicio físico utilizado dentro de la rehabilitación cardiaca contribuyó a disminuir la grasa visceral, lo cual se evidenció en los $3,4 \mathrm{~cm}$ de disminución en el perímetro de la cintura encontrados al final del programa. Además de este, el incremento en los valores de la capacidad cardiorrespiratoria confirmó que un programa de ejercicios planeado y estructurado con base en una evaluación sistemática posibilita un incremento de la capacidad funcional de los sujetos postcirugía de la revascularización miocárdica - la angioplastia coronaria percutánea y en consecuencia mejora su participación en las actividades de la vida diaria. El cambio obtenido en los parámetros antropométricos y en la capacidad funcional mejoran el pronóstico de la enfermedad coronaria y disminuyen los factores de riesgo metabólico a corto plazo.

\section{Responsabilidades éticas}

Protección de personas y animales. Los autores declaran que los procedimientos seguidos se conformaron a las normas éticas del comité de experimentación humana responsable y de acuerdo con la Asociación Médica Mundial y la Declaración de Helsinki

Confidencialidad de los datos. Los autores declaran que han seguido los protocolos de su centro de trabajo sobre la publicación de datos de pacientes.

Derecho a la privacidad y consentimiento informado. Los autores han obtenido el consentimiento informado de los pacientes y/o sujetos referidos en el artículo. Este documento obra en poder del autor de correspondencia.

\section{Conflicto de intereses}

Ninguno de los autores manifiesta conflicto de interés en el presente estudio.

\section{Reconocimientos y agradecimientos}

Los autores reconocen y agradecen a la Universidad Simón Bolívar y a la Fundación del Caribe para la Investigación Biomédica por apoyar la presente investigación. Igualmente, a todos los pacientes que voluntariamente manifestaron su consentimiento para hacer parte del estudio.

\section{Bibliografía}

1. Gómez JE. Morbimortalidad cardiovascular en el mundo. Rev. Colomb Cardiol. 2012;19:298-9.

2. Gaziano TA, Bitton A, Anand S, Abrahams GS, Murphy A. Growing epidemic of coronary heart disease in low-and middle-income countries. Curr. Probl. Cardiolog. 2010;35:72-115.
3. Jelinek HF, Huang ZQ, Khandoker AH, Chang D, Kiat H. Cardiac rehabilitation outcomes following a 6-week program of $\mathrm{PCl}$ and CABG patients. Front Physiol. 2013;4:1-7.

4. Burdiat G. Rehabilitación cardiaca después de un síndrome coronario agudo. Rev. Urug. Cardiol. 2014;29:153-63.

5. Aragam KG, Dai D, Neely ML, Bhatt DL, Roe MT, Rumsfeld JS, et al. Gaps in referral to cardiac rehabilitation of patients undergoing percutaneous coronary intervention in the United States. J Am Coll Cardiol. 2015;65:2079-88.

6. García Al, Pereira JE. Tolerancia al ejercicio en pacientes cardiovasculares posquirúrgicos luego de la intervención con un programa de rehabilitación cardiaca fase II. Rev. Colomb. Cardiol. 2014;21:409-13.

7. Goel K, Lennon RJ, Tilbury RT, Squires RW, Thomas RJ. Impact of cardiac rehabilitation on mortality and cardiovascular events after percutaneous coronary intervention in the community. Circulation. 2011;123:2344-52.

8. Vanhees L, Rauch B, Piepoli M, Van Buuren F, Takeen T, Borjesson $M$, et al. Importance of characteristics and modalities of physical activity and exercise in the management of cardiovascular health in individuals with cardiovascular disease (Part III) Eur. J. Prev. Cardiol. 2012;19:1333-56.

9. Dayan V, Ricca R. Rehabilitación cardiaca luego de la cirugía de revascularización miocárdica. Arch. Cardiol. Mex. 2014;84:286-92.

10. Mezzani A, Hamm L, Jones AM, McBride PE, Moholdth T, Stone JA, et al. Aerobic exercise intensity assessment and prescription in cardiac rehabilitation: a joint position statement os the European Association for Cardiovascular Prevention And Rehabilitation, the American Association of Cardiovascular and Pulmonary Rehabilitation and the Canadian Association of Cardiac Rehabilitation. Eur. J. Prev. Cardiol. 2013;20: 442-67.

11. Conraads VM, Pattyn N, De Meyer C, Beckers PS, Coeckelberghs E, Cornelissen VA, et al. Aerobic interval training and continuos training equally improve aerobic exercise capacity in patients with coronary artery disease: the SAINTEX-CAD study. Int. J. Cardiol. 2015;179:203-10.

12. Sumide T, Shimada, Ohmura K, Onishi H, Kawakami T, Masaki $\mathrm{K}$, et al. Relationship between exercise tolerance and muscle strength following cardiac rehabilitation: comparison of patients after cardiac surgery and patients with myocardial infarction. J. Cardiol. 2009;54:273-81.

13. Kwan G, Balady GJ. Cardiac rehabilitation 2012 advancing the field through emerging science. Circulation. 2012;125:369-73.

14. Márquez J, Suárez G, Márquez J. Beneficios del ejercicio en la insuficiencia cardiaca. Rev. Chil. Cardiol. 2013;32:58-65.

15. Gallo J, Saldarriaga J, Clavijo M, Arango E, Rodríguez N, Osorio $\mathrm{J}$, et al. Actividad física y salud cardiovascular: en búsqueda de la relación dosis respuesta. 1 th ed. Medellín (Antioquia): Corporación para Investigaciones Biológicas (CIB); 2010.

16. Tehortúa DS, Gallo JA, Rico M, Durango L. Efecto de un programa de rehabilitación cardiaca basado en el ejercicio sobre la capacidad física, la función cardiaca y la calidad de vida, en pacientes con falla cardiaca. Rev. Colom. Cardiol. 2011;18:25-36.

17. López F, Pérez C, Zeballos PC, Anchique CV, Burdiat G, González $\mathrm{K}$, et al. Consenso de rehabilitación cardiovascular y prevención secundaria de las sociedades interamericana y sudamericana de cardiología. Rev. Urug. Cardiol. 2013;28:189-224.

18. Alberti K, Eckel RH, Grundy SM, Zimmet PZ, Cleeman JI, Donato KA, et al. Harmonizing the metabolic Syndrome A Joint Interim Statement of the International Diabetes Federation Task Force on Epidemiology and Prevention; National Heart, Lung and Blood Institute; American Heart association; World heart Federation; International Atherosclerosis Society; and international Association for the Study of Obesity. Circulation. 2009;120:1640-5. 
19. Adedoyin RA, Adeyaniu SA, Balogun MO, Adebayo RA, Akintomide AO, Akinwusi PO. Prediction of funtional capacity during six-minute walk among patients with chronic heart failure. Niger J ClinPract. 2010;13:379-81.

20. Lizarzaburu JC. Síndrome metabólico: concepto y aplicación práctica. An. Fac. Med. 2013;74:315-20.

21. Aschner P, Buendía R, Brajkovich I, González A, Figueredo R, Juarez XE, et al. Determination of the cutoff point for waist circumference that establishes the presence of abdominal obesity in Latin American men and women. Diabetes Res. Clin. Pract. 2011;93:243-7.

22. Nalini M, Moradi B, Esmaeilzadeh M, Maleki M. Does the effect of supervised cardiac rehabilitation programs on body fat distribution remained long time? J. Cardiovasc. Thoracic. Res. 2013;5:133-8.

23. Valencia CA, Jiménez OJ, Díaz ML, Mazadiego ML. Correlación entre la escala de Borg modificada y la saturación de oxígeno durante la prueba de esfuerzo máxima en pacientes postinfartados. Rev. Mex. Med. Fis. Rehab. 2012;24: 5-9.

24. Mancia G, Fagard R, Narkiewicz K, Redon J, Zanchetti A, Bohm M, et al. Guía de práctica clínica de la ESH/ESC para el manejo de la hipertensión arterial [2013]. Hipertens. Riesgo Vasc. 2013;30:4-91.

25. Camargo DM, Campos MT, Sarmiento JM, Garzón M, Navia JJ, Merchán A. Respuesta hemodinámica con el entrenamiento en resistencia y fuerza muscular de los miembros superiores en la rehabilitación cardiaca. Rev. Col. Cardiol. 2007;14:198-206.

26. Aikawa P, Cintra A, Oliveira J, Silva C, Pierucci J, Afonso M, et al. Reabilitacao cardiaca em pacientes submetidos a cirurgia de revascularizacao do miocardio. Rev. Bras. Med. Esporte. 2014;20:55-8.

27. Heran BS, Chen JM, Ebrahim S, Moxham T, Oldridge N, Rees K, et al. Exercise-based cardiac rehabilitation for coronary heart disease. Cochrane Database Syst. Rev. 2011;6:1-128.

28. Najafi F, Nalini M. Hospital-based versus hybrid cardiac rehabilitation program in coronary bypass surgery patients in western Iran: effects on exercise capacity, risk factors, psychological factors, and quality of life. J. Cardiopulm. Rehabil. Prev. 2015;35:29-36.

29. Taylor RS, Dalal H, Jolly K, Zawada A, Dean SG, Cowie A, et al. Home-based versus centre-based cardiac rehabilitation. Cochrane Database Syst. Rev. 2015;8:CD007130, http://dx.doi.org/ 10.1002/14651858.CD007130.pub3. 